\title{
Основные факторы эффективности в поведенческих теориях лидерства
}

\author{
Радюк О.М. ${ }^{1}$, Басинская И.В. ${ }^{1}$, Нехвядович И.Г. ${ }^{1}$ \\ 1 Белорусский государственный университет, Минск, Республика Беларусь
}

\begin{abstract}
АННОТАЦИЯ:
Научная новизна статьи обусловлена эволюционным подходом авторов к развитию теорий лидерства, в котором выделяются четыре этапа: 1) теория лидерских качеств, 2) поведенческие теории лидерства, 3) ситуационные теории лидерства, 4) интеракционистские теории лидерства. Поведенческие теории лидерства, которым посвящена настоящая статья, являются логичным продолжением теории лидерских качеств и закономерным предшественником ситуационных теорий лидерства. В статье подробно рассмотрены поведенческие модели лидерства К.Левина (авторитарный, демократический и попустительский стили лидерства), Д. Хемфилла и Э. Кунса (структурирование и внимательность), Р. Кана и Д. Каца (ориентация на работников и ориентация на производство). Показано, что, несмотря на различия в терминологии, все поведенческие модели лидерства указывают на два основных фактора, связанных с эффективностью лидерства: поведение, ориентированное на достижения, и поведение, ориентированное на межличностные взаимоотношения. Обсуждены ограничения поведенческих моделей, которые впоследствии привели исследователей к созданию ситуационных теорий лидерства.
\end{abstract}

КЛючЕВЫЕ СЛОВА: теории лидерства, теория лидерских качеств, поведенческие теории лидерства, ситуационные теории лидерства, интеракционистские теории лидерства, поведение лидера, стили лидерства

\section{Key performance factors in behavioral theories of leadership}

Radyuk O.M. ${ }^{1}$, Basinskaya I.V. ' , Nekhvyadovich I.G. ${ }^{1}$

${ }^{1}$ Belarusian State University, Belarus

\section{Введение}

сследователи приступили к изучению поведенческих аспектов
лидерства в 1940-х годах - после того, как теория лидерских качеств потерпела временное фиаско. Изучение поведения представлялось не столь сложным, как изучение личностных особенностей: по сравнению со свойствами характера, поведение легче наблюдать, его проще измерить, и ему можно научить [19] (Кuhn, 2001). Исследователи поставили перед собой задачу с помощью наблюдения за поведением установить, что именно делают эффективные лидеры и как они это делают [4] (Bass, 1990). 
Поведенческие аспекты лидерства изучались в трех университетах США - в Университете штата Айова (Курт Левин и соавт.), Университете штата Огайо (Джон Хемфилл, Элвин Кунс) и Мичиганском университете (Роберт Кан, Даниил Кац) - каждый из которых сформулировал свою модель лидерства. Рассмотрим их подробнее.

\section{Модель К. Левина: авторитарный, демократический и попустительский стили лидерства}

Т зучая особенности взаимодействия лидеров с последователями, исследователи университета Айовы Курт Левин с соавторами в 1939 году выделили авторитарный, демократический и попустительский стили лидерства [12, с. 180; 20] (Ghosh,2000; Lewin, Lippitt, White, 1939).

Авторитарные лидеры обычно занимают доминирующее положение в разговоре, дают задания своим последователям и стараются контролировать их с помощью законов и предписаний $[10,21,25]$ (Day, Hamblin, 1964; Ley, 1966; Shaw, 1955). Авторитарное лидерство способно повысить продуктивность, когда лидер находится на своем рабочем месте, однако такой стиль лидерства часто приводит к высокой теку-

\section{ABSTRACT:}

The scientific novelty of the article lies in the evolutionary approach of the authors to the development of the theory of leadership, in which the authors mark four stages such as 1) theory of leadership skills, 2) behavioral theories of leadership, 4) situational leadership theories, 4) interactionist theories of leadership. The article is devoted to the behavioral theories of leadership, which are a logical continuation of the theory of leadership qualities and the logical precursor of situational leadership theories. The article examines in detail behavior models of leadership of K.Lewin lauthoritarian, democratic and passive leadership styles), D. Hemphill and E. Koons (structuring and mindfulness), R. Kahn and D. Katz (orientation towards workers and orientation towards production). The article shows that despite the differences in terminology, all behavioral models of leadership point out two main factors related to leadership effectiveness such as performance-oriented behavior and interpersonal relationshiporiented behavior. We consider the limitations of behavioral models, which later led researchers to creation of situational leadership theories.

KEYWORDS: theories of leadership, theory of leadership skills, behavioral theories of leadership, situ ational leadership theories, interactionist theories of leadership, behavior of a leader, leadership styles.

JEL Classification: L26, M12, M54

Received: 20.01.2017 / Published: 30.06.2017

(C) Author(s) / Publication: CREATIVE ECONOMY Publishers

For correspondence: Radyuk O.M. (6311141amail.ru)

\section{CITATION:}

Radyuk O. M., Basinskaya I. V., Nekhvyadovich I. G. (2017) Osnovnye faktory effektivnosti v povedencheskikh teoriyakh liderstva [Key performance factors in behavioral theories of leadership]. Liderstvo $i$ menedzhment. 4. (2). - 99-107. doi: 10.18334/lim.4.2.38063 
чести персонала и может формировать у сотрудников агрессивное отношение к организации [13] (Hackman, Johnson, 2013).

Демократические лидеры более отзывчивы и менее зависимы от законов и предписаний, чем авторитарные. Демократические лидеры делегируют полномочия своим подчиненным, которые легко находят общий язык со своими лидерами. Демократический стиль лидерства способствует повышению удовлетворенности работников [22] (Mohr, 1971), приверженности решениям лидера [28] (Ziller, 1957), участию [16] (Hespe G., Wall, 1976) и новаторству [11] (Farris, 1972), одновременно уменьшая отток персонала и количество невыходов на работу [3] (Argyle, Gardner, Cioffi,1958).

Попустительский (англ. «laissez-faire») стиль лидерства, иногда именуемый в отечественных публикациях «пассивным» [2] (Filonovich, 1999), характеризуется склонностью лидера уходить от ответственности и перекладывать бремя принятия решения на своих подчиненных. Такие лидеры стремятся свести взаимодействие с подчиненными к нулю, делегируя им все свои права и полномочия и позволяя последователям поступать так, как им хочется.

Попустительский стиль лидерства, как правило, уменьшает удовлетворенность подчиненных и их вовлеченность в работу [5] (Baumgartel, 1957). Однако в группе, состоящей из высокомотивированных экспертов, такой стиль лидерства, напротив, увеличивает удовлетворенность работников и повышает их продуктивность [26] (Weschler, Kahane, Tannenbaum, 1952).

\section{Модель Д. Хемфилла и Э. Кунса: структурирование и внимательность}

Т вое исследователей из университета штата Огайо, Джон Хемфилл и Элвин Кунс, в 1959 году опубликовали результаты своих многолетних исследований особенностей лидерского поведения [14, 15] (Hemphill, Coons, 1957; Hemphill, Coons, 1957). Первоначально Д. Хемфилл и Э. Кунс рассматривали более 1000 потенциальных независимых характеристик поведения лидеров, но постепенно сократили их коли-

\section{ОБ АВТОРЕ:}

Радюк Олег Михайлович, доцент кафедры общей и клинической психологии БГУ, кандидат медицинских наук, доцент (6311141वmail.ru)

Басинская Ирина Валентиновна, старший преподаватель кафедры общей и клинической психологии БГУ (3075007amail.ru)

Нехвядович Ирина Гаврииловна, специалист управления кадров БГУ, магистр психологии (nehvyadovichabsu.by)

\section{ЦИТИРОВАТЬ СТАТЬЮ:}

Радюк О. М., Басинская И. В., Нехвядович И. Г. Основные факторы эффективности в поведенческих теориях лидерства // Лидерство и менеджмент. - 2017. - Том 4. - № 2. - С. 99-107. doi: 10.18334/lim.4.2.38063 
чество до двух: структурирования (англ. "initiating structure") и внимательности (англ. “consideration") [1, c. 226; 24] (Robbinz, 2006; Nelson, Quick, 2012).

Структурирование относится к активности, сфокусированной на достижении поставленных целей, такой как определение функциональных обязанностей и рабочих отношений, определение структуры и организация выполнения работы. Лидер с высоким уровнем структурирования умеет распределить задания между подчиненными, требует неукоснительного соблюдения установленных стандартов качества, следит за своевременностью выполнения порученных подчиненным заданий.

Внимательность же в данной модели обозначает поведение, сфокусированное на построении уважительных взаимоотношений с подчиненными. Лидер с высоким уровнем внимательности выстраивает с подчиненными основанные на взаимном доверии и уважении равноправные рабочие отношения, беспокоится о благополучии, статусе и комфорте подчиненных, помогает им решать их персональные проблемы, всегда дружелюбен и стремится к эффективной коммуникации.

В процессе выполнения данной работы авторами был создан «Опросник описания поведения лидера» (англ. "Leader Behavior Description Questionnaire”, LBDQ).

Как указывают авторы модели, структурирование и внимательность являются не столько двумя полюсами одной шкалы, сколько двумя различными континуумами. Т.е. лидер может проявлять 1) один из этих двух типов лидерского поведения, 2) оба типа, либо 3) ни одного.

Лидеры, поведение которых характеризуется одновременно и структурированием, и внимательностью, чаще добиваются высокой производительности от своих подчиненных и обеспечивают их большую удовлетворенность работой, но так происходит далеко не всегда, поскольку воздействие структурирования и внимательности могут быть нейтрализованы рядом ситуационных факторов, которые не учитываются поведенческими моделями лидерства [1, с. 226; 24] (Robbinz, 2006; Nelson, Quick, 2012). Попытка учесть это воздействие была предпринята впоследствии Робертом Хаузом в ситуационной модели «путь - цель» [17] (House, Mitchell, 1974).

\section{Модель Р. Кана и Д. Каца: ориентация на работников и ориентация на производство}

П очти одновременно с исследователями университета Огайо, в 1960 году, опу1 бликовали результаты своего исследования лидерского поведения и сотрудники Исследовательского центра опросов общественного мнения при Мичиганском университете Роберт Кан и Даниил Кац [18]. Мичиганские исследователи также пришли к выводу о существовании двух основных характеристик поведения лидеров, которые они назвали терминами «ориентация на работников» (англ. “employee_oriented") и «ориентация на производство» (англ. "production_oriented”).

Первоначально Р. Кан и Д. Кац предполагали, что существует единый континуум 
стилей лидерства с двумя противоположенными полюсами «ориентация на работников» и «ориентация на производство». Однако после досконального изучения поведения лидеров они выделили два независимых континуума [18] - так же, как это было сделано в исследовании университета штата Огайо.

Лидеры, ориентированные на производство, стремятся к выполнению поставленных перед ними задач любой ценой. К своим подчиненным они относятся лишь как к «человеческим ресурсам», необходимым для достижения цели. Лидеры, ориентированные на работников, уделяют большое внимание межличностным отношениям, они искренне интересуются своими подчиненными и демонстрируют индивидуальный подход при работе с каждым из них. Мичиганские исследователи сделали вывод о предпочтительности ориентации на работников, поскольку она обеспечивала более высокие уровни удовлетворенности работой и производительности труда [1, с. 227] (Robbinz, 2006).

Выявленные исследователями Мичиганского университета два ключевых параметра поведения лидеров - «ориентация на работников» и «ориентация на производство» - легли в основу модели «управленческой решетки», которую Роберт Блейк и Джейн Мутон предложили вниманию общественности в 1969 году [6-8] (Blake, Mouton, 1969; Blake, Mouton, 1994; Blake, Mouton, 1972). Расположение этих параметров в Декартовой системе координат позволило выделить широкий спектр (всего 81) лидерских стилей.

Авторы модели полагали, что наиболее эффективным является стиль «командное управление», характеризующийся наивысшими уровнями обоих параметров как ориентации на работников, так и ориентации на производство. Но сохраняет ли данное утверждение свою истинность в меняющихся контекстах? И существуют ли вообще универсальные (не зависящие от ситуаций) эффективные стили лидерства? Эти вопросы полны разногласий и остаются нерешенными по сей день [27] (Yukl, 2012).

\section{Выводы}

Т зучение поведенческих моделей лидерства показало, что, несмотря на различия в терминологии, все они указывают на два основных фактора, связанных с эффективностью лидерства: «ориентация на достижения» и «ориентация на межличностные взаимоотношения» [9] (Campbell, 1991). Поведение, ориентированное на достижения (англ. “task achievement”), включает в себя структурирование группы, организацию работы, определение и достижение целей, планирование и управление [9] (Campbell, 1991). Поведение, ориентированное на межличностные взаимоотношения (англ. “interpersonal relations”), направлено на формирование взаимного доверия, уважения и заботы (англ. “trust, respect and concern”) и включает в себя такие виды активности, как делегирование полномочий, создание альянсов с коллегами, руководителями и 
принимающими решения лицами, активное слушание и получение обратной связи, эффективная коммуникация, убеждение, мотивирование, воодушевление и конфронтация [9] (Campbell, 1991).

Названия, используемые для обозначения указанных факторов в моделях различных исследовательских групп, представлены в таблице.

Таблица

Названия факторов, связанных с эффективностью лидерства, в различных моделях в рамках поведенческих теорий

\begin{tabular}{|c|c|c|c|}
\hline \multirow[b]{2}{*}{ Авторы модели } & \multicolumn{3}{|c|}{ Факторы } \\
\hline & $\begin{array}{c}\text { Ориентация } \\
\text { на достижения }\end{array}$ & $\begin{array}{c}\text { Ориентация } \\
\text { на межличностные } \\
\text { взаимоотношения }\end{array}$ & Иные факторы \\
\hline $\begin{array}{l}\text { Курт Левин с соавторами } \\
\text { [20] (Lewin, Lippitt, White, } \\
\text { 1939) }\end{array}$ & $\begin{array}{l}\text { Авторитарный } \\
\text { стиль лидерства }\end{array}$ & $\begin{array}{l}\text { Демократический стиль } \\
\text { лидерства }\end{array}$ & $\begin{array}{l}\text { Попустительский } \\
\text { стиль лидерства }\end{array}$ \\
\hline $\begin{array}{l}\text { Джон Хемфилл и Элвин } \\
\text { Кунс [14, 15] (Hemphill, } \\
\text { Coons, 1957; Hemphill, } \\
\text { Coons, 1957) }\end{array}$ & Структурирование & Внимательность & - \\
\hline $\begin{array}{l}\text { Роберт Кан и Даниил } \\
\text { Кац [18], Роберт Блейк } \\
\text { и Джейн Мутон [6-8] } \\
\text { (Blake, Mouton, 1969; } \\
\text { Blake, Mouton, 1994; } \\
\text { Blake, Mouton, 1972) }\end{array}$ & $\begin{array}{l}\text { Ориентация на } \\
\text { производство }\end{array}$ & $\begin{array}{l}\text { Ориентация на работ- } \\
\text { ников }\end{array}$ & - \\
\hline
\end{tabular}

Источник: составлено авторами

Хотя в отдельных исследованиях утверждалось, что наилучшие результаты дает сочетание ориентации на достижения с ориентацией на межличностные взаимоотношения, устойчивые взаимосвязи между этими характеристиками поведения лидера и результатами работы возглавляемой лидером группы так и не были обнаружены [1, c. 228] (Robbinz, 2006).

Различные лидеры, демонстрирующие идентичное поведение, далеко не всегда достигают одинаковой эффективности. Более того, один и тот же лидер, проявляющий одно и то же поведение в двух разных ситуациях, не всегда одинаково эффективен в обоих контекстах. Это осознание впоследствии привело исследователей к необходимости изучения ситуативных переменных и созданию ситуационных теорий пидерства [23] (Nahavandi, 2012). 


\section{ИСТОЧНИКИ:}

1. Роббинз С.П. Основы организационного поведения. / Монография. - Москва: Вильямс, 2006. - 448 с.

2. Филонович С.Р. Лидерство и практические навыки менеджера: 17-модульная программа для менеджеров «Управление развитием организации». / Модуль 9. - М.: ИНФРА-М, 1999. - 328 c.

3. Argyle M., Gardner G., Cioffi F. Supervisory methods related to productivity, absenteeism, and labour turnover // Human Relations. - 1958. - p. 23-40.

4. Bass, B.M. From Transactional to Transformational Leadership / B.M. Bass. - 1990. - 3. - 19-31 p.

5. Baumgartel H. Leadership Style as a Variable in Research Administration // Administrative Science Quarterly. - 1957. - № 3. - p. 344-360.

6. Blake R.R., Mouton J.S. Building a dynamic corporation through grid organization development. - MA: Addison-Wesley Educational Publishers Inc, 1969. - 128 p.

7. Blake R.R., Mouton J.S. The Managerial Grid. - Houston, Texas: Gulf Publishing, 1994. $-350 \mathrm{p}$.

8. Blake R.R., Mouton J.S. The Managerial Grid: Key Orientations For Achieving Production Through People. - Houston, Texas: Gulf Publishing Company, 1972. - 340 p.

9. Campbell D.P. Manual for the Campbell Leadership Index. - Minneapolis, MN: National Computer System, 1991.

10. Day R.C., Hamblin R.L. Some Effects of Close and Punitive Styles of Supervision // American Journal of Sociology. - 1964. - № 5. - p. 499-510.

11. Farris G.F. The effect of individual roles on performance in innovative groups // R\&D Management. - 1972. - № 1. - p. 23-28.

12. Ghosh B. Human Resource Development and Management. - New Delhi: Sangam Books Ltd, 2000. $-376 \mathrm{p}$.

13. Hackman M.Z., Johnson C.E. Leadership: A Communication Perspective, Sixth Edition. - Long Grove, Illinois: Waveland Press, Inc., 2013. - 530 p.

14. Hemphill J., Coons A. Development of the leader behavior description and measurement // Columbus: Business Research, Ohio State University. - 1957. - p. 1-18.

15. Hemphill J.K., Coons A.E. Development of the leader behavior description questionnaire // Leader behavior: Its description and measurement. - 1957. - № 6. - p. 38.

16. Hespe G., Wall T. The Demand for Participation among Employees // Human Relations. - 1976. - № 5. - p. 411-428.

17. House R.J., Mitchell T.R. Path-goal theory of leadership // Journal of Contemporary Business. - 1974. - № 3. - p. 81-97.

18. Kahn, R.L. Leadership Practices in Relation to Productivity and Morale / R.L. Kahn, D. Katz // Group Dynamics, Research and Theory / eds. D. Cartwright, A. Zander. - New York: Harper \& Row, 1968. 
19. Kuhn Jr T.M. The relationship between employee personality traits and preferred leadership style. - Las Vegas: University of Nevada, 2001. - 89 p.

20. Lewin K., Lippitt R., White R.K. Patterns of Aggressive Behavior in Experimentally Created “Social Climates" // Journal of Social Psychology. - 1939. - № 2. - p. 269-299.

21. Ley R. Labor turnover as a function of worker differences, work environment, and authoritarianism of foremen // Journal of Applied Psychology. - 1966. - № 6. - p. 497500 .

22. Mohr L.B. Organizational Technology and Organizational Structure // Administrative Science Quarterly. - 1971. - № 4. - p. 444-459.

23. Nahavandi A. The Art and Science of Leadership. - New York: Pearson Education, 2012. $-417 \mathrm{p}$.

24. Nelson D.L., Quick J.C. Organizational Behavior: Science, The Real World, and You. Mason, OH: South-Western College Pub, 2012. - 800 p.

25. Shaw M.E. A comparison of two types of leadership in various communication nets // Journal of Abnormal and Social Psychology. - 1955. - № 1. - p. 127-134.

26. Weschler I.R., Kahane M., Tannenbaum R. Job satisfaction, productivity and morale: a case study // Occupational Psychology. - 1952. - № 26. - p. 1-14.

27. Yukl G.A. Leadership in Organizations. - New York: Pearson Education, 2012. - 528 p.

28. Ziller R.C. Four techniques of group decision making under uncertainty // Journal of Applied Psychology. - 1957. - № 6. - p. 384-388.

\section{REFERENCES:}

Argyle M., GardnerG., Cioffi F. (1958). Supervisory methods related to productivity, absenteeism, and labour turnover Human Relations. 11 23-40.

Baumgartel H. (1957). Leadership Style as a Variable in Research Administration Administrative Science Quarterly. 2 (3). 344-360.

Blake R.R., Mouton J.S. (1969). Building a dynamic corporation through grid organization development MA: Addison-Wesley Educational Publishers Inc.

Blake R.R., Mouton J.S. (1972). The Managerial Grid: Key Orientations For Achieving Production Through People Houston, Texas: Gulf Publishing Company.

Blake R.R., Mouton J.S. (1994). The Managerial Grid Houston, Texas: Gulf Publishing. Campbell D.P. (1991). Manual for the Campbell Leadership Index Minneapolis, MN: National Computer System.

Day R.C., Hamblin R.L. (1964). Some Effects of Close and Punitive Styles of Supervision American Journal of Sociology. 69 (5). 499-510.

Farris G.F. (1972). The effect of individual roles on performance in innovative groups R\&D Management. 3 (1). 23-28. 
Filonovich S.R. (1999). Liderstvo i prakticheskie navyki menedzhera: 17-modulnaya programma dlya menedzherov «Upravlenie razvitiem organizatsii» [Leadership and practical skills of the manager: a 17-module program for managers «Management of the development of the organization»] M.: INFRA-M. (in Russian).

Ghosh B. (2000). Human Resource Development and Management New Delhi: Sangam Books Ltd.

Hackman M.Z., Johnson C.E. (2013). Leadership: A Communication Perspective, Sixth Edition Long Grove, Illinois: Waveland Press, Inc.

Hemphill J., Coons A. (1957). Development of the leader behavior description and measurement Columbus: Business Research, Ohio State University. 1-18.

Hemphill J.K., Coons A.E. (1957). Development of the leader behavior description questionnaire Leader behavior: Its description and measurement. (6). 38.

Hespe G., Wall T. (1976). The Demand for Participation among Employees Human Relations. 29 (5). 411-428.

House R.J., Mitchell T.R. (1974). Path-goal theory of leadership Journal of Contemporary Business. (3). 81-97.

Kuhn Jr T.M. (2001). The relationship between employee personality traits and preferred leadership style Las Vegas: University of Nevada.

Lewin K., Lippitt R., White R.K. (1939). Patterns of Aggressive Behavior in Experimentally Created "Social Climates" Journal of Social Psychology. 10 (2). 269299.

Ley R. (1966). Labor turnover as a function of worker differences, work environment, and authoritarianism of foremen Journal of Applied Psychology. 50 (6). 497-500.

Mohr L.B. (1971). Organizational Technology and Organizational Structure Administrative Science Quarterly.16 (4). 444-459.

Nahavandi A. (2012). The Art and Science of Leadership New York: Pearson Education.

Nelson D.L., Quick J.C. (2012). Organizational Behavior: Science, The Real World, and You Mason, OH: South-Western College Pub.

Robbinz S.P. (2006). Osnovy organizatsionnogo povedeniya [Basic Concepts of Organisational Behaviour] Moscow: Vilyams. (in Russian).

Shaw M.E. (1955). A comparison of two types of leadership in various communication nets Journal of Abnormal and Social Psychology. 50 (1). 127-134.

Weschler I.R., Kahane M., Tannenbaum R. (1952). Job satisfaction, productivity and morale: a case studyOccupational Psychology. (26). 1-14.

Yukl G.A. (2012). Leadership in Organizations New York: Pearson Education.

Ziller R.C. (1957). Four techniques of group decision making under uncertainty Journal of Applied Psychology.41 (6). 384-388. 